\title{
Computer Aided Detection of Dental Implant Crown Defects
}

\author{
V.G. BÖCEKÇI* AND Ö. DEMIR \\ Department of Electric and Electronics Engineering, Faculty of Technology, Marmara University, \\ 34722 İstanbul, Turkey
}

\begin{abstract}
A dental implant is an artificial tooth root that is placed into to hold a replacement tooth or bridge. Crown is the apparent part of the dental implant. Detection of crown defects is important for health of the patient before the surgery. In this study, a computer aided detection system developed to analyze tooth dental implant crown digital images to detect defects. The developed system detects various implant defects such as fractures, cracks, dents, spots and roughness. Micro fractures are detected using morphologic operations by the system. Dents and spots are also detected using this image processing techniques. Textural analysis can be performed on digital images to detect different roughness and porosity areas on dental implant. The developed system prevents waste of time and decreases costs. Using the system also will increase patients' satisfaction.
\end{abstract}

DOI: 10.12693/APhysPolA.134.422

PACS/topics: structural failure of materials, image analysis, image processing algorithms

\section{Introduction}

Biomaterials are materials that are identified as including living systems systematically and pharmacologically [1]. Biomaterials are also commonly used in dentistry. Biomaterials are developed over time in the field of the dentistry. Metal-based materials are replaced with ceramic types due to biocompatibility and corrosion [2]. There are significant challenges in the monitoring and displaying of biomaterials tissues and structures. For this reason, various imaging techniques are developed to struggle these challenges [3]. Digital photography is the most inexpensive imaging method. Besides of the its cost, receiving image and transmitting to a computer is easy, rapid and no need too much experience. In the near future, imaging is exactly digitized with regards to contemporary prosthesis techniques. The development of digital technology will play an important role in the monitoring the movement of the jaw, prosthesis manufacturing and dental education. Digital image processing, pattern analysis and artificial intelligence techniques have application areas such as prosthesis odontotherapy, orthodontia and oral surgery that are increasing day by day. Some companies are making efforts to develop new hardware and software in these areas [4].

The aim of this study is developing a computer aided detection (CAD) system to detect defects that can be occurred in dental prosthesis produced for patients by using image processing techniques. In this study, irregularities such as spots, cracks, fractures on dentures and also problems that can affect the quality of the usage are detected by image processing techniques. Detection of such defects by an expert is a difficult process. For this reason, the detection of these defects by the developed system improves the quality of the production.

\footnotetext{
*corresponding author; e-mail: vgbocekci@marmara.edu.tr
}

\section{Materials and method}

Developed CAD system consists of three phases. In the preprocessing phase, RGB images are converted to gray level images and enhanced. In the segmentation phase, images are separated into meaningful regions for detection. In the detection phase, defects of the tooth image are detected and labeled. Block diagram of the CAD system is presented in Fig. 1.

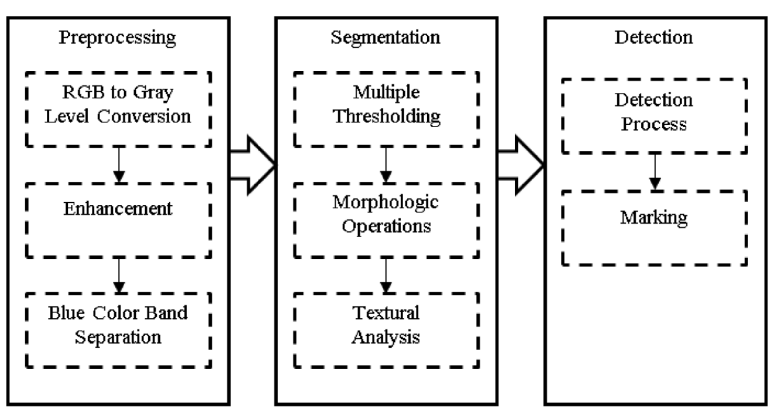

Fig. 1. Block diagram of the developed system.

\subsection{Preprocessing phase}

Digital camera images of the dental crown images are true color RGB images. In preprocessing phase, RGB images are converted to gray level intensity images. The obtained dental crown images are digital true color RGB images. In order to use some image processing techniques more effectively, these images are converted to gray-level images. Thus, only the luminance effect appears on the images while the effects of hue and saturation are eliminated.

\subsection{Segmentation phase}

The term image segmentation refers to the partition of an image into a set of regions. Aim of the segmentation is for the regions to represent meaningful areas of 
the image. The regions might be sets of border pixels grouped into such structures as line segments or circular segments. Regions may also be defined as groups of pixels having both a border and a particular shape such as a circle or polygon [5]. Image segmentation is the process of separating target regions in a digital image from others. These targeted areas, which are meaningful for the purpose of image processing application, can be geometric shapes such as line, circle, polygon, or struct Äares without a certain shape. The pixel values between these two threshold values constitute the regions of interest, in which defects may be present [5-7]. An original image matrix and thresholded image matrix with minimum threshold value 100 , maximum threshold value 180 are shown in Fig. 2a,b. Then, thresholded images are converted into binary images.

(a)
\begin{tabular}{|c|c|c|c|c|c|c|c|c|c|c|c|c|c|c|c|c|c|c|}
\hline 147 & 147 & 147 & 145 & 164 & 163 & 110 & 84 & 84 & 83 \\
\hline 145 & 147 & 147 & 146 & 163 & 161 & 112 & 83 & 82 & 81 \\
\hline 143 & 175 & 176 & 178 & 178 & 183 & 112 & 82 & 81 & 81 \\
\hline 139 & 173 & 174 & 176 & 178 & 182 & 115 & 78 & 81 & 81 \\
\hline 138 & 172 & 173 & 174 & 179 & 182 & 117 & 73 & 78 & 80 \\
\hline 130 & 133 & 160 & 170 & 173 & 144 & 117 & 67 & 147 & 147 & 145 & 164 & 163 & 0 & 0 & 0 & 0 \\
\hline 143 & 175 & 176 & 176 & 163 & 161 & 0 & 0 & 0 & 0 \\
\hline 130 & 132 & 165 & 169 & 172 & 143 & 129 & 65 & 717 & 77 & 176 & 178 & 178 & 180 & 179 & 0 & 0 & 0 \\
\hline 0 & 172 & 173 & 174 & 179 & 180 & 0 & 0 & 0 & 0 \\
\hline 0 & 0 & 160 & 170 & 173 & 144 & 0 & 0 & 0 & 0 \\
\hline 130 & 132 & 143 & 147 & 158 & 143 & 129 & 64 & 68 & 69 \\
\hline 130 & 110 & 99 & 87 & 79 & 74 & 70 & 63 & 62 & 60 & 165 & 169 & 172 & 0 & 0 & 0 & 0 & 0 \\
\hline 0 & 0 & 143 & 147 & 0 & 0 & 0 & 0 & 0 & 0 \\
\hline 0 & 0 & 0 & 0 & 0 & 0 & 0 & 0 & 0 & 0 \\
\hline
\end{tabular}

Fig. 2. Image matrixes: (a) original image matrix, (b) the thresholded matrix.

(a) (b \begin{tabular}{|c|c|c|c|c|c|c|c|c|c|c|c|c|c|c|c|c|c|c|c|}
\hline 0 & 0 & 0 & 1 & 1 & 0 & 0 & 0 \\
\hline 0 & 1 & 0 & 0 & 0 & 0 & 1 & 1 \\
\hline 1 & 1 & 1 & 0 & 0 & 1 & 1 & 1 \\
\hline 1 & 1 & 1 & 1 & 0 & 1 & 1 & 1 \\
\hline 0 & 0 & 0 & 0 & 1 & 1 & 0 & 0 & 0 & 0 & 0 & 0 \\
\hline 0 & 1 & 1 & 0 & 0 & 1 & 1 & 1 \\
\hline 0 & 1 & 0 & 0 & 0 & 0 & 1 & 0 \\
\hline 0 & 0 & 0 & 0 & 0 & 0 & 0 & 0 \\
\hline 1 & 1 & 1 & 0 & 0 & 1 & 1 & 1 \\
\hline 1 & 1 & 1 & 1 & 0 & 1 & 1 & 1 \\
\hline 1 & 0 & 0 & 1 & 1 & 1 & 0 & 0 & 0 & 0 & 0 & 0 & 0 & 0 \\
\hline 0 & 1 & 1 & 0 & 0 & 0 & 0 & 1 & 1 \\
\hline 1 & 1 & 1 & 0 & 0 & 1 & 1 & 1 \\
\hline 1 & 1 & 1 & 1 & 0 & 1 & 1 & 1 \\
\hline 0 & 1 & 0 & 0 & 0 & 0 & 1 & 1 \\
\hline & 0 & 0 & 0 & 0 & 0 & 0 & 0 \\
\hline & 0 & 0 & 1 & 1 & 1 & 0 & 0 & 1 & 1 & 1 \\
\hline 0 & 1 & 0 & 0 & 0 & 0 & 1 & 0 \\
\hline 0 & 0 & 0 & 0 & 0 & 0 & 0 & 0 \\
\hline 0 & 0 & 0 & 0 & 0 & 0 & 0 & 0 \\
\hline
\end{tabular}

Fig. 3. Binary matrixes: (a) binary matrix, (b) detected neighborhoods on binary matrix, (c) detected neighborhoods after the elimination.

The technique of conducting an adjacency examination on binary images is frequently used to identify abnormal forms in the numerous image processing applications. A procedure can be performed on the actual image using the coordinates of the identified forms on the binary image as a result of the adjacency examination. Conducting an adjacency examination on binary images involves the identification of values of 1 present on adjacent pixels. For all pixels with a value of 1 , the adjacencies of every pixel in all 8 directions are evaluated; this process is repeated for the every adjacent pixel with a value of 1. When the adjacency examination is performed for a minimum number of adjacencies, some forms which are smaller than the neighborhood of 4 pixels are ignored in order to simplify the calculation $[5,8,9]$. A binary matrix is shown in Fig. 3a, detected all neighborhoods on the binary matrix are shown in Fig. 3b, in Fig. 3c detected forms are marked without ignored forms.

Textural analysis of the dental image performed to identify different surface characteristics such as porosity and roughness. The gray level co-occurrence matrices (GLCM) are useful for the textural analysis on 2D images. GLCM supplies second order statistical features from pixel values of a texture image. GLCM is dependent on directional information of neighbor pixel values. Calculation of a GLCM is presented in Fig. 4a and b. Five different features are extracted from GLCM to identify different textural areas on dental image. Dental image is separated into $9 \times 9$ areas for textural analysis. A GLCM is calculated for the each area and the five features are extracted. Each feature vector is recorded as a row of a feature matrix. After a correlation investigation of feature matrix, areas with similar textural characteristic are labeled on dental image using a color map. Extracted features from a GLCM are listed below:

Contrast

$$
\sum_{i=0}^{G-1} \sum_{j=0}^{G-1} \operatorname{Co}(i, j)(i-j)^{2}
$$

Energy

$$
\sum_{i=0}^{G-1} \sum_{j=0}^{G-1} \operatorname{Co}(i-j)^{2}
$$

Entropy

$$
\sum_{i=0}^{G-1} \sum_{j=0}^{G-1}-\mathrm{Co}(i, j) \log (\mathrm{Co}(i, j))
$$

Homogeneity

$$
\sum_{i=0}^{G-1} \sum_{j=0}^{G-1} \frac{1}{1+(i-j)^{2}} \operatorname{Co}(i, j)
$$

Momentum

$$
\sum_{i=0}^{G-1} \sum_{j=0}^{G-1} \frac{(\operatorname{Co}(i, j))^{2}}{1+|i-j|}
$$

In the equations, $G$ represents row and column number of the GLCM, $i$ and $j$ represent row and column indices,

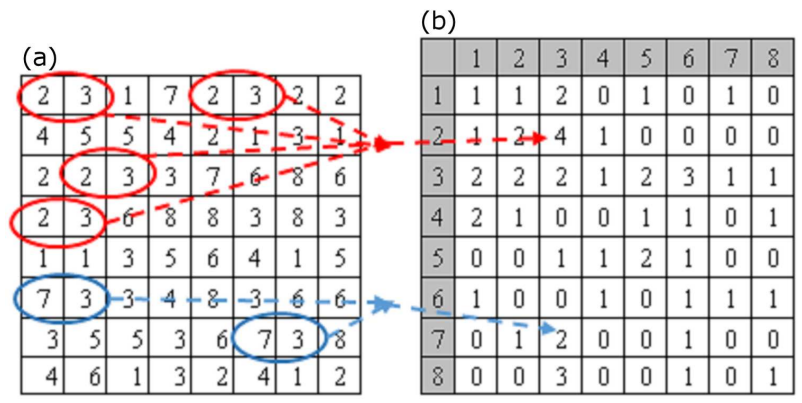

Fig. 4. Calculation of the GLCM: (a) image matrix, (b) calculated GLCM according to pixel values. 
$\mathrm{Co}(i, j)$ represents an element of the GLCM. Areal rates of different type areas are calculated according to correlation results. The regions with similar textural characteristics are marked with the same color.

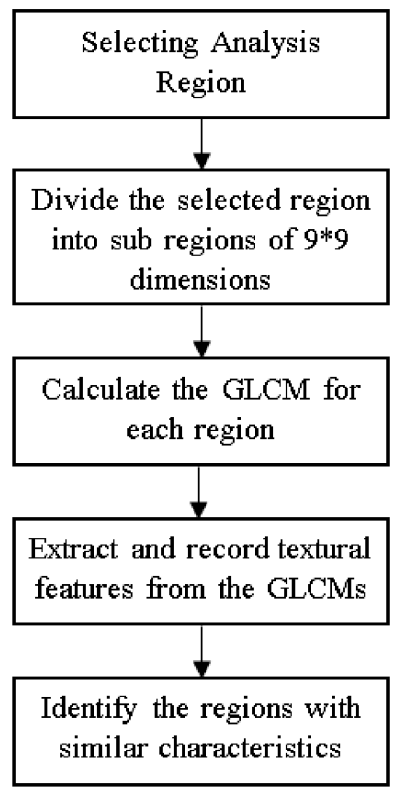

Fig. 5. Flow chart of the semi-automatic texture analysis process.

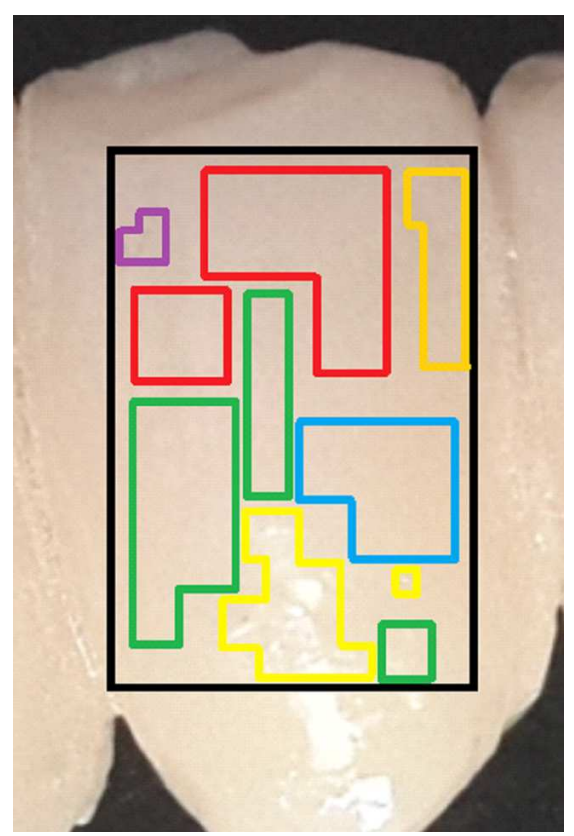

Fig. 6. Textural analysis result of a dental image.

Texture analysis is implemented semi-automatically on a selected area by the CAD system. Performing the texture analysis on the entire image causes unnecessary and meaningless results because of the lighting problems. Flow chart of the semi-automatic texture analysis process is presented in Fig. 5. Textural analysis results of a dental image is shown in Fig. 6.

\subsection{Detection phase}

After preprocessing and segmentation phase, dental implant crown defects can be detected. Detection phase of the developed system is able to detect different types of defects such as cracks, fractures, dents, and spots. For fractures and cracks detection, morphologic structure elements are used. Morphologic dilation method is used to detect dents. Blue color band separation and connected component labeling methods are used for spot detection.

\subsection{Experiments with the CAD system}

Thirty different digital dental implant crown images have been used in experiments. These images includes fractures, cracks, dents, spots, and surface problems. The experimental results showed that the CAD system is successful on crack, fracture, dent, and spot detection. Also the CAD system detects different surface characteristics and color differences using semi-automatic textural analysis. The CAD system was unable to distinguish similar color differences when full automatic textural analysis was performed.

\section{Results and discussion}

Developed system is successful on detection of dental implant crown defects. Cracks, fractures, dents, spots and surface problems are detected by developed system. Steps of a fracture detection are presented in Fig. 7. Detection of a dent process is shown in Fig. 8. In Fig. 9, spot detection process steps are presented.
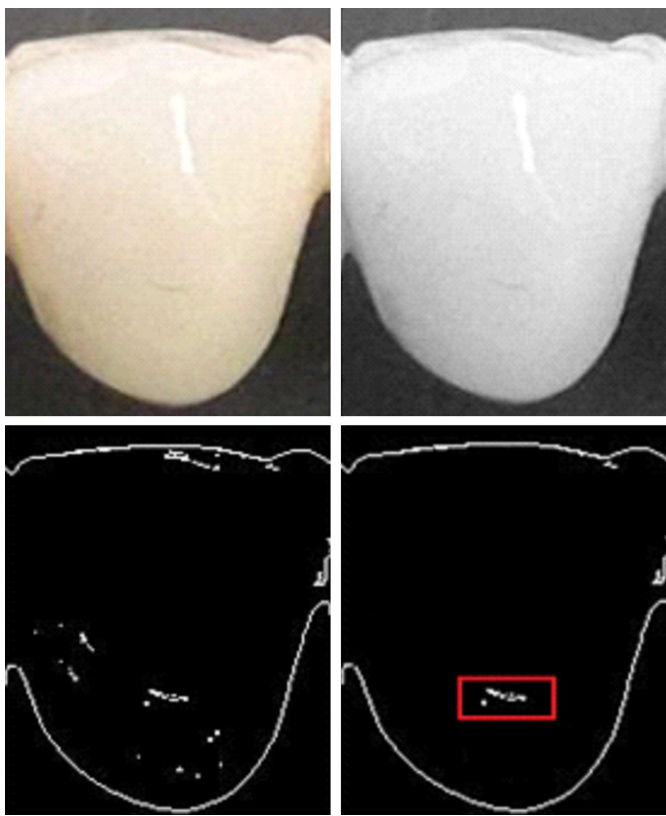

Fig. 7. Steps of a fracture detection process. 


\section{Conclusion}

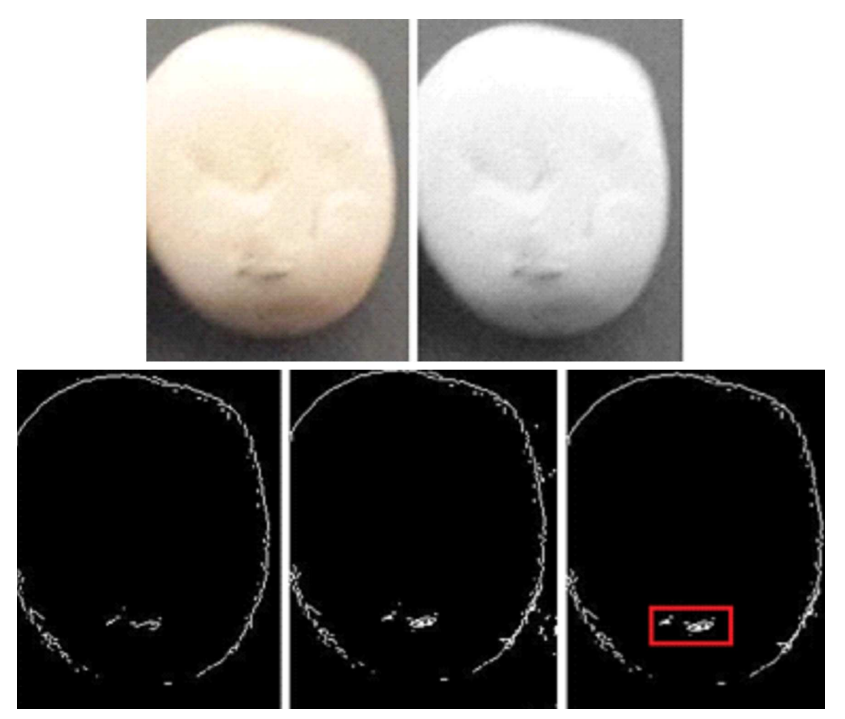

Fig. 8. Steps of a dent detection .

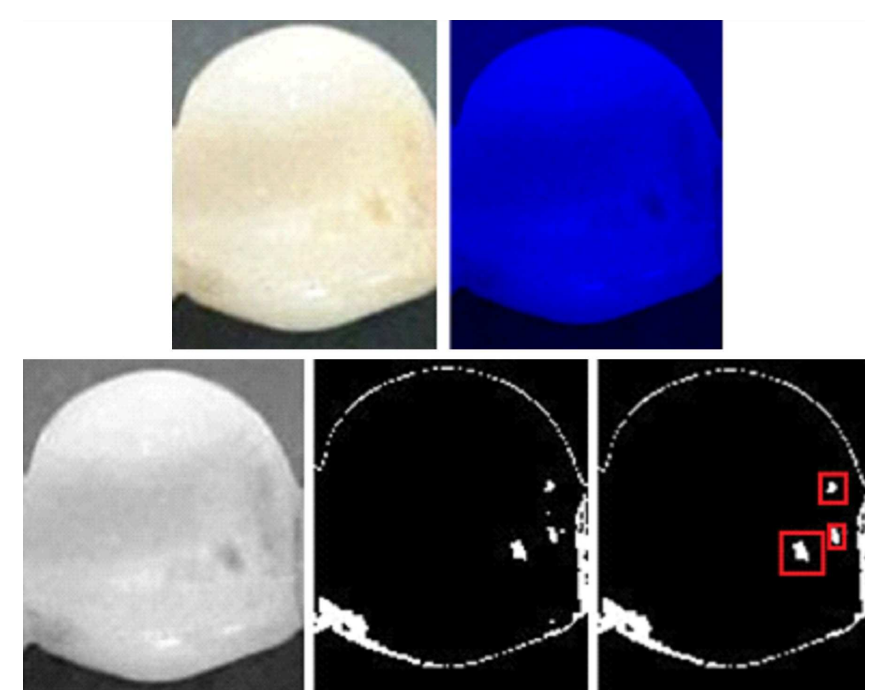

Fig. 9. Steps of spots detection .
In this study, a CAD system is developed to detect surface defects of dental implant crowns. Crack, fracture, dent, and spot defects of crown surfaces can be detected easily before the implementation. The system also detects surface problems using textural analysis. The ability of the system to work on digital photography images reduces costs. By using the system, waste of time can be prevented and implementation costs are decreased. In addition, patient's satisfaction can be increased.

\section{References}

[1] J.B. Park, Biomaterials Science and Engineering, Springer Science \& Business Media, 2012.

[2] V. Migonney, A. Mainjot, Biomaterials, 2014, p. 181.

[3] A.A. Appel, M.A. Anastasio, J.C. Larson, E.M. Brey, J. Biomaterials 34.28, 6615 (2013).

[4] R. Bhambhani, J. Bhattacharya, S.K. Sen, J. Indian Prosthodont Soc. 13, 165 (2013).

[5] O. Demir, A.Y. Camurcu, Biomed. Mater. Eng. 26, $1213(2015)$

[6] M. Sezgin, B. Sankur, J. Electron. Imag. 13, 146 (2004).

[7] N. Otsu, IEEE Trans. Syst. Man Cyb. 9, 62 (1979).

[8] P.A. Devijver, Connected Components in Binary Images: The Detection Problem, Wiley, New York 1984.

[9] L.D. Stefano, A. Bulgarelli, in: Proc. 10th Int. Conf. on Image Analysis and Processing, 1999, p. 322 .

[10] R.M. Haralick, Proc. IEEE 67, 786 (1979).

[11] R.M. Haralick, K. Shanmugam, I.H. Dinstein, IEEE Trans. Syst. Man Cyb. 3, 610 (1973).

[12] X. Xie, Electron. Lett. Comp. Vis. Im. An. 7, 1 (2008).

[13] L.S. Davis, M. Clearman, J.K. Aggarwal, in: Proc. 18th IEEE Conf. on Decision and Control, Vol. 1, 1979, p. 71. 